\title{
Oral Health Knowledge and Sources of Information Among Elementary Schoolchildren
}

\author{
Marilyn W. Woolfolk, DDS, MPH* \\ Assistant Professor \\ W. Paul Lang, DDS, MPH \\ Associate Professor \\ Barbara Wirth Faja, MPH \\ Research Associate \\ Department of Prevention and Health Care \\ School of Dentistry. University of Michigan \\ Ann Arbor, M! 48109-1078
}

ing levels of oral health knowledge in subgroups of the population then becomes desirable.

An early attempt to assess existing oral health knowledge of school-aged children was conducted by Linn in 1969 (2). This survey of tenth graders in Minnesota found that the teenagers were "sorely lacking" in fundamental knowledge about what is needed for optimum oral health. These adolescents were least knowledgeable about periodontal disease. Some five years later, Henderson et al. (3) surveyed a group of Iowa children and adolescents in grades 1-12 and concluded that concepts of preventive dentistry were not well known among the population. Children were asked about preventive dentistry in a limited context, however, as only knowledge about the cause of tooth decay and the use of dental floss and disclosing tablets were investigated.

Prior to establishing a school-based dental health education program, Walsh (4) measured the dental health knowledge of 854 boys and girls aged 12-14 years in San Francisco. Finding that fewer than 50 percent of the respondents correctly answered eight of ten questions about dental caries and periodontal disease, she concluded that prevailing knowledge was inadequate. B. A. Russell and A. M. Horowitz (unpublished observations) compared the knowledge of a sample of sixth graders who had completed a four year regimen of the National Preventive Dentistry Demonstration Program (NPDDP) and a similarly aged control group that did not participate in the program. No differences were found between the groups with regard to knowledge of fluoride use and its benefits. Students who had received sealants from the program were somewhat more knowledgeable about the purpose of sealants.

While the preceding investigations vary with regard to the age groups surveyed and the types of knowledge assessed, the inadequacy of children's dental knowleage is apparent. Still, an incomplete picture of oral health awareness remains, as most of these surveys evaluated understanding of disease risk factors and not recognition of efficacious preventive agents like fluoride and fissure sealants. Sources of oral health information for adults have been examined $(5,6)$, but docu-
* Send correspondence and reprints to Dr. Woolfolk. Manuscript received: 4/28/88; returned to author for revision: 6/3/88; accepted for publication: $6 / 20 / 88$. 
mentation of children's sources has been limited. The sources most frequently reported by children participating in the NPDDP were the school and the dental office (Russell BA and Horowitz AM, unpublished observations). Clearly, more inquiry into children's sources of health information is needed. Examining schoolchildren's knowledge of dental disease and preventive agents was the focus of this study. Additionally, children's sources of information about oral health were determined and the relations between knowledge and a child's age, sex, caries severity, and sources of information were investigated.

\section{Methods}

In 1985, a clinical toothpaste trial commenced in southwestern Michigan. Examinations were performed on 2,036 children at 23 elementary schools. The children resided in 46 communities in seven counties. Table 1 displays some selected demographic parameters of the area. Median income levels for the seven counties ranged from $\$ 17,503$ to $\$ 22,211$. The percent of persons that were high school graduates in these counties varied from a low of 62 percent to a high of 76 percent (7). Generally, these parameters were similar to state and national averages. Persons-per-dentist ratios in the seven counties ranged from 1,562:1 to 4,950:1 (8), representing a spectrum of availability of dental professionals.

A convenience sample of 848 fourth- and sixth-grade schoolchildren was selected for the present study. All subjects were participating in the clinical trial and were examined for decayed, missing, and filled permanent tooth surfaces (DMFS). Assessment of caries was based on criteria reported by Radike (9). A sealant was considered to be present if it could be detected clinically, regardless of whether the pits or fissures were completely or partially covered. Subjects were examined while seated in a portable dental chair by one examiner (WPL) using a mouth mirror and No. 5 explorer under illumination.

To determine levels of oral health knowledge, a questionnaire was developed. Because the children were at different grade levels, it was necessary to design the questionnaire to be readable and understandable for the youngest children. Some questions were modeled after the American Dental Association (ADA) curriculum guide for teachers (10). Few items in the ADA
TABLE 1

Demographic Characteristics of Study Area, State and National Comparisons (7)

\begin{tabular}{lccc}
\hline Area & $\begin{array}{c}\text { \% High } \\
\text { School } \\
\text { Graduates }\end{array}$ & $\begin{array}{c}\text { \% Below } \\
\text { Poverty } \\
\text { Level }\end{array}$ & $\begin{array}{c}\text { Median } \\
\text { Family } \\
\text { Income (\$) }\end{array}$ \\
\hline County & & & \\
Branch & 65.3 & 10.7 & 18,535 \\
Cass & 63.4 & 11.0 & 18,548 \\
Hillsdale & 68.7 & 10.8 & 17,503 \\
Jackson & 69.2 & 9.3 & 21,363 \\
Kalamazoo & 75.8 & 10.7 & 22,211 \\
St. Joseph & 65.7 & 9.7 & 18,609 \\
$\quad$ VanBuren & 62.1 & 13.5 & 17,700 \\
Michigan & 68.0 & 10.4 & 22,107 \\
United States & 66.5 & 12.4 & 19,917 \\
\hline
\end{tabular}

guide focused on preventive agents, so additional questions were devised to determine knowledge levels about fissure sealants and fluoride. Pretesting early drafts of the survey instrument on groups of fourthand sixth-grade schoolchildren resulted in adjustments of the instrument length, wording, and phrasing of questions, as it became clear that completion of the questionnaire could be problematic for some children. Thus, the final questionnaire was shortened to $11 \mathrm{mul}$ tiple-choice questions having brief responses, including "I don't know." Questions focused on knowledge of dental disease, sclected preventive measures, and children's soucces of information. All questionnaires were completed under supervision in the classroom.

Frequency distributions of all responses were generated. A knowledge scale was then constructed based upon numbers of correct responses. Respondents were stratified into groups by level of knowledge: low (less than three answers correct), medium (four to six correct), and high (seven or more correct). To assess relations between knowledge and caries severity, DMFS scores were also stratified into low (caries-free), medium (one to five DMFS), and high (greater than five DMFS) categories. Decayed (D) and filled (F) components of the index were similarly stratified. Chisquared statistics were then performed to determine the relation of age, sex, and caries severity to knowl-

TABLE 2

Distribution of Sample by Age, Sex, and Mean DMFS Scores

\begin{tabular}{|c|c|c|c|c|c|c|c|c|}
\hline \multirow[b]{2}{*}{ Age } & \multicolumn{2}{|c|}{ Males } & \multicolumn{2}{|c|}{ Females } & \multicolumn{2}{|c|}{ Total } & \multirow{2}{*}{$\begin{array}{l}\text { Mean } \\
\text { DMFS }\end{array}$} & \multirow[b]{2}{*}{ SD } \\
\hline & $\%$ & $n$ & $\%$ & $n$ & 7 & $n$ & & \\
\hline 9 years & 26.3 & (106) & 31.9 & $(142)$ & 29.2 & $(248)$ & 1.19 & 0.98 \\
\hline 10 years & 25.3 & (102) & 19.3 & $(86)$ & 22.2 & $(188)$ & 1.60 & 1.42 \\
\hline 11 years & 24.8 & $(100)$ & 32.6 & (145) & 28.9 & $(245)$ & 2.08 & 1.41 \\
\hline 12 years & 23.6 & (95) & 16.2 & $(72)$ & 19.7 & (167) & 2.42 & 1.93 \\
\hline All ages & 100.0 & (403) & 100.0 & $(445)$ & 100.0 & $(848)$ & 1.78 & 1.45 \\
\hline
\end{tabular}


edge, and the relation between extent of knowledge and source of information.

\section{Results}

In Table 2, the distribution of the sample of age, sex, and mean DMFS scores is displayed. The children ranged in age from nine to 12 years, females representing a slightly larger proportion than males. The mean DMFS score for the group was 1.78; 48 percent were caries free. Of those children completing the questionnaire, 46 individuals exhibited at least one sealant on a permanent tooth.

The children's responses to questions about caries prevention (Table 3 ) suggested varying degrees of recognition of these concepts. Seventy-five percent of respondents correctly identified the action of fluoride as preventing decay, yet only 4 percent of the children knew that the best source of fluoride was the water supply: 61 percent of the children selected fluoridated toothpaste, while another 23 percent chose fluoride application by the dentist. Although about one-quarter of the respondents were able to correctly define a dental sealant, 46 percent of the children answered "I don't know." Of the children with sealants, 50 percent of

TABLE 3

Distribution of Responses to Questions about Caries Prevention

\begin{tabular}{|c|c|c|}
\hline Question & $n$ & $\%$ \\
\hline \multicolumn{3}{|l|}{ What does fluoride do? } \\
\hline 1. It makes teeth white. & 144 & 17.1 \\
\hline * 2. It helps protect teeth from decay. & 630 & 74.9 \\
\hline 3. It makes teeth grow. & 10 & 1.2 \\
\hline 4. I don't know. & 57 & 6.8 \\
\hline \multicolumn{3}{|l|}{ The best way to get fluoride is to: } \\
\hline $\begin{array}{l}\text { 1. Have a dentist put fluoride on your } \\
\text { teeth. }\end{array}$ & 197 & 23.4 \\
\hline $\begin{array}{l}\text { 2. Brush your teeth with a fluoride } \\
\text { toothpaste. }\end{array}$ & 515 & 61.2 \\
\hline * 3. Drink water that has fluoride in it. & 36 & 4.3 \\
\hline 4. I don't know. & 93 & 11.1 \\
\hline \multicolumn{3}{|l|}{ What is a dental sealant? } \\
\hline $\begin{array}{l}\text { 1. A coating that keeps gums from } \\
\text { bleeding. }\end{array}$ & 58 & 6.9 \\
\hline $\begin{array}{l}\text { * 2. A plastic coating put on teeth to } \\
\text { protect them from decay. }\end{array}$ & 196 & 23.3 \\
\hline 3. A kind of tooth filling. & 202 & 24.0 \\
\hline 4. I don't know. & 384 & 45.7 \\
\hline \multicolumn{3}{|l|}{ What snack foods are best for teeth?t } \\
\hline 1. Raisins & 674 & 79.9 \\
\hline 2. Candy bars & 21 & 2.5 \\
\hline * 3. Popcorn & 190 & 22.5 \\
\hline * 4. Cheese & 774 & 91.7 \\
\hline 5. Ice cream & 185 & 21.9 \\
\hline *6. Nuts & 419 & 49.6 \\
\hline
\end{tabular}

* Correct answer

tMore than one response allowed.

Percents may not add to $100 \%$ due to rounding these individuals correctly defined a sealant, compared to only 22 percent of the remaining children (chisquared test, $P<.01$ ). The children demonstrated a limited ability to differentiate among snack foods that could potentially affect the health of their teeth.

Replies to questions about periodontal health are found in Table 4. Most respondents could define plaque, could identify a sign of gum disease, and recognized that the best way to keep from getting gum disease was to clean their mouths every day. Also, 89 percent of the children recognized dental floss as a cleaning device for between the teeth. The specifics of plaque removal were apparently less well understood, as 34 percent of the children thought that plaque should be removed "only by a dentist."

The distribution of knowledge levels by age is shown in Figure 1. A higher proportion of the younger children (nine and ten years of age) demonstrated low levels of knowledge when compared with the older children. The 11-year-old group contained the highest proportion $(82 \%)$ of students with medium and high levels of knowledge. While older children tended to have higher levels of knowledge, that relation was not found to be significant using chi-squared statistics.

\section{TABLE 4}

Distribution of Responses to Questions about Periodontal Health

\begin{tabular}{lrr}
\hline Question & $n$ & $\%$ \\
\hline What is plaque? & 12 & 1.4 \\
1. A toothpaste. & 792 & 93.6 \\
* A layer of germs on the teeth. & 20 & 2.4 \\
3. A plastic coating for teeth. & 22 & 2.6 \\
4. I don't know. & 65 & 7.7 \\
Blood on your toothbrush may be a sign of: & & \\
1. Plaque. & 598 & 70.7 \\
2. Gum disease. & 89 & 10.5 \\
3. Tooth decay. & 94 & 11.1 \\
4. I don't know. & &
\end{tabular}

The best way to keep from getting gum disease is:

1. Eat a good diet. $\quad 414.8$

* 2. Clean your mouth every day. $\quad 720 \quad 84.9$

3. Take vitamins. $\quad 35 \quad 4.1$

4. I don't know. $\quad 52 \quad 6.1$

Plaque should be removed:

* 1. At least once a day. $\quad 496 \quad 58.6$

2. Only by a dentist. $\quad 290 \quad 34.2$

3. Never.

4. I don't know.

0.5

The best way to clean between your teeth is to:

1. Use a toothbrush.

8.8

* 2. Use dental floss.

3. Use a toothpick.

89.1

4. I don't know.

0.5

*Correct answer.

Percents may not add to $100 \%$ due to rounding. 
FIGURE 1

Distribution of Children's Knowledge Levels by Age

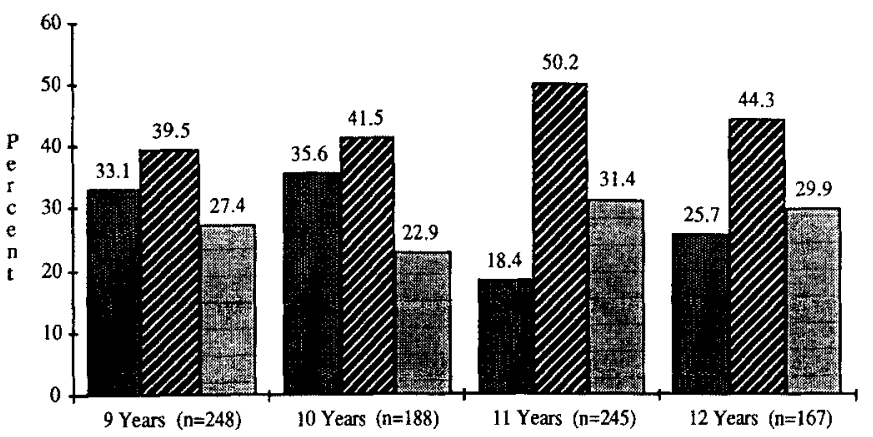

\% Low Level $\boldsymbol{\square}$ \% MediumLevel $\%$ High Level

FIGURE 2

Sources of Information about Oral Health

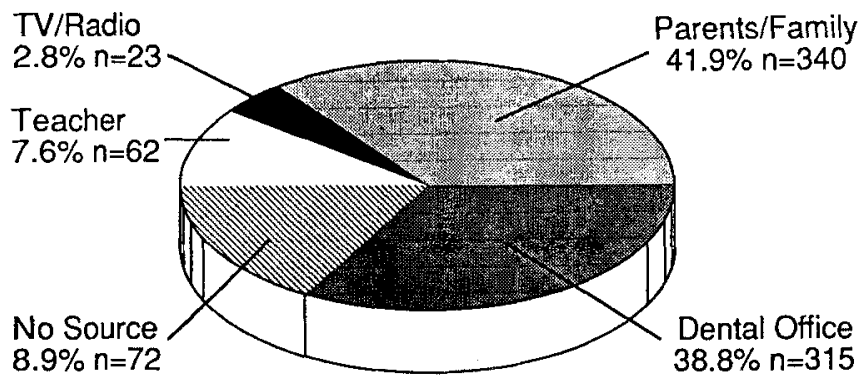

FIGURE 3

Distribution of Children's Knowledge Levels by Source of Information

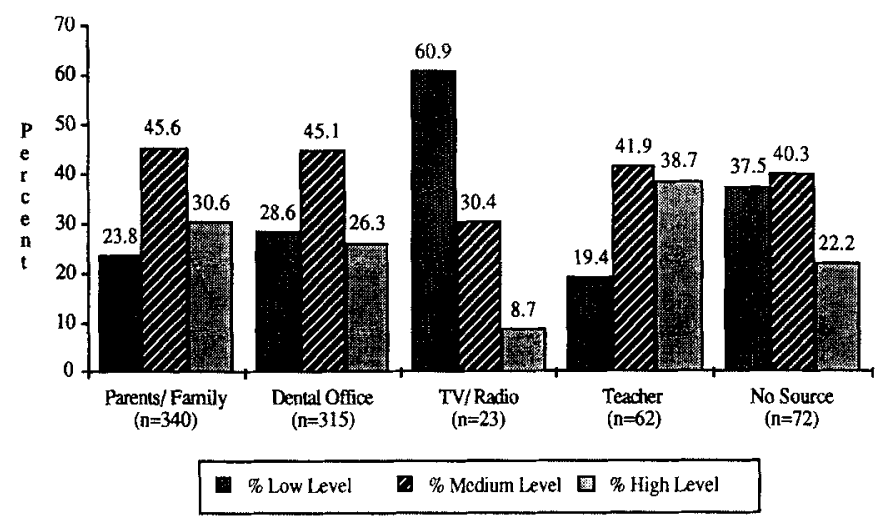

Knowledge level was not found to be related to sex, nor to levels of DMFS scores nor to decayed and filled components of the scores.

Figure 2 highlights the reported sources of children's information about oral health. Forty-two percent of the children indicated that parents and family members taught them the most about dental health and caring for their teeth. Thirty-nine percent of respondents cited "someone at the dentist's office." Other sources of in- formation-teachers, media (TV, radio)-were reported by few children.

The extent of correct information possessed by the children varied by reported source (Figure 3); but no statistically significant relation was found between source of information and extent of knowledge. Children who answered the most questions correctly were likely to name parents or family as a source of information and then the dentist's office. A comparison of these two sources showed minimal differences in the levels of correct information. Although teachers were cited by a relatively small number of children, almost 40 percent of those students demonstrated high levels of knowledge and another 42 percent demonstrated medium levels of oral health knowledge. The group of students that reported media as a source of information demonstrated the least knowledge. Paradoxically, among children who did not cite a particular source, 63 percent displayed a medium or high level of correct information.

\section{Discussion}

This study did not find any relation between levels of knowledge and age, sex, caries severity, or children's sources of information. A positive relation between filled teeth and knowledge and a negative relation between decayed teeth and knowledge have been reported by Sgan-Cohen and coworkers (11); however, children in that investigation were older: 15 years of age. Such associations may not have manifested themselves in the current study because the children were young, and nearly half the population was caries free.

Some observations about schoolchildren's knowledge can be made. Regarding prevention of caries, most children in the study correctly identified the fact that fluoride protects teeth from caries. Yet most of the respondents were unaware that the fluoride in drinking water is the most effective means of affording protection. Apparently, the relative effectiveness of different fluoride vehicles had not been learned by this group.

Most children were unable to define a dental sealant. This discovery parallels observations of Russell and Horowitz, who found that only 21 percent of the sixth graders who had not participated in the NPDDP knew the purpose of sealants (Russell BA and Horowitz AM, unpublished observations). Even among the individuals with sealants in the present study, only 50 percent of these children could define a sealant. This finding suggests that during sealant placement, dental professionals should seize the opportunity to educate the public about this preventive agent.

With respect to periodontal health, children were more successful at defining plaque, recognizing a sign of gum disease, and identifying the best way to prevent gum disease. Awareness of periodontal disease seems to have increased among children over the past ten years when findings are compared to Linn's results (2). In that report, few children knew periodontal disease was a disease of the gingiva, and there was no evidence that they knew about plaque. However, in the present 
study, while many children correctly defined plaque, more than one-third of the children were confused about plaque removal. The children may have equated plaque removal with tartar removal. Recent advertising of tartar control products might explain their uncertainty as to whether plaque should be removed "only by a dentist."

Analysis of sources of information revealed that parents and family represented the children's primary source of information about oral health. The children's knowledge may then reflect the disappointing realities of parental and adult awareness of the value of fluorides and fissure sealants. An opinion study (5) comparing attitudes about dental health among dental researchers, practitioners, and the public found that the public thinks about fluoride infrequently and in limited situations. Indeed, data from a nationwide survey of adults conducted by the National Center for Health Statistics (NCHS) in 1985 revealed that only 45 percent of adults surveyed in the United States perceived drinking fluoridated water to be "definitely important" in preventing decay, and only 18 percent of the adults had heard of, and knew the purpose of, dental sealants (12). From a historical perspective, this situation is understandable. The addition of fissure sealants to the preventive armamentarium has proceeded slowly, and this service has not been widely promoted by dental professionals (13). Parents are not familiar with the use of sealants from their own dental experience; most likely they are unsure of the appropriateness of these procedures for their child unless recommended by the dental profession (14).

The optimal way to raise children's dental health awareness would be to furnish accurate information to parents. The role of the dental office must be acknowledged, however, as this source. was cited by a large number of children. Furthermore, two-thirds of adults responding to a national survey conducted by $\mathrm{O}^{\prime}$ Neill (5) reported that they rely upon their dentists for information and considered the dental profession as a very credible source. In many situations, an adult family member may act as an intermediary between the dental office and the child. Dental professionals, then, have the responsibility to interpret, clarify, and correct information that an adult or child may gather from other sources and to identify effective preventive strategies that are appropriate for that individual. This responsibility perhaps necessitates a purposeful shift away from emphasizing oral hygiene procedures exclusively to introducing and reinforcing knowledge of other preventive strategies. The low levels of knowledge about sealants and fluoridated water discovered among children also may be the result of the limited emphasis placed on these preventive methods by dental professionals. Knowledge levels of children who reported media as a source suggest that this manner of acquiring correct information may not be particularly effective, although the small sample size makes such an interpretation speculative. Findings indicate that teachers may be a promising source of information for children; however, further investigation of information sources is merited.
Regardless of the children's recognized source of information, some critical gaps in knowledge are apparent. Concerns raised by Corbin and coworkers (12) are indicative of the dilemma faced by public health workers: "If this suboptimal and inconsistent pattern of knowledge about oral diseases and their control is not corrected, effective disease prevention efforts on both an individual and a community level will be limited." Not correcting knowledge patterns bodes ill for future fluoridation efforts, as well. A recent incident in a community in the study area underscores the need for providing correct information to the public. A referendum on continuation of water fluoridation was lost by ten votes (15). Such a loss indicates that public health advocates cannot ignore education of the community. This occurrence reinforces the need to "develop meaningful and effective health education messages addressed to all people, old and young alike" (12). Dental professionals and public health workers must continue to emphasize the risk factors related to dental diseases and the importance of fluoridation and fissure sealants in controlling caries. It is through these efforts that progress toward future national health objectives can be realized.

\section{References}

1. US Department of Health and Human Services. Public Health Service. Promoting health/preventing disease. Objectives for the Nation. Washington, DC: Government Printing Office, 1980:54.

2. Linn EL. Teenagers' attitudes, knowledge, and behaviors related to oral health. J Am Dent Assoc 1976 May;92:946-51.

3. Henderson WG, Skeele DK, Soule DJ. A sample survey of dental knowledge, attitudes, behavior and needs among Scott County school children. Iowa Dent J 1974 Aug;60:14-6.

4. Walsh MM. Effects of school-based dental health education on knowledge, attitudes and behavior of adolescents in San Francisco. Community Dent Oral Epidemiol 1985 June;13:143-7.

5. O'Neill HW. Opinion study comparing attitudes about dental health. I Am Dent Assoc 1984 Dec;109:910-15.

6. Lang WP, Faja BW, Woolfolk MW, Glasrud PH, Frazier PJ. Elementary schoolteachers' knowledge and attitudes about oral health [Abstract]. J Dent Res 1987 Mar;66(Spec Iss):299.

7. US Bureau of the Census. County and city data book, 1983. Washington DC: Government Printing Office, 1983

8. Michigan Department of Public Health. Michigan health statistics. Lansing: Michigan Department of Public Health, 1984.

9. Radike AW. Criteria for diagnosis of dental caries. In: Proceedings of the Conference on the Clinical Testing of Cariostatic Agents. Chicago: American Dental Association, 1968:87-8.

10. American Dental Association. Learning about your oral health. A prevention-oriented school program. Level II:4-6. Chicago: American Dental Association, 1973:1-17.

11. Sgan-Cohen HD, Lipsky R, Behar R. Caries, diet, dental knowledge and socioeconomic variables in a population of 15 -year-old Israeli schoolchildren. Community Dent Oral Epidemiol 1984 Oct;12:332-6

12. Corbin SB, Mass WR, Kleinman DV, Backinger CL. 1985 NHIS findings on public knowledge and attitudes about oral diseases and preventive measures. Public Health Rep 1987;102:53-60.

13. Cohen L, Labelle A, Romberg E. The use of pit and fissure sealants in private dental practice. J Public Health Dent 1988 Winter; $48(1): 26-35$

14. Lang WP, Weintraub JA, Choi C, Bagramian RA. Fissure sealant knowledge and characteristics of parents as a function of their child's sealant status. J Public Health Dent 1988 Summer;48:133-7.

15. Board of Canvassers. Canvass of votes cast at the election held on March 9, 1987, Kalamazoo County, MI. Kalamazoo, MI: Doubleday Bros \& Co., 1987. 